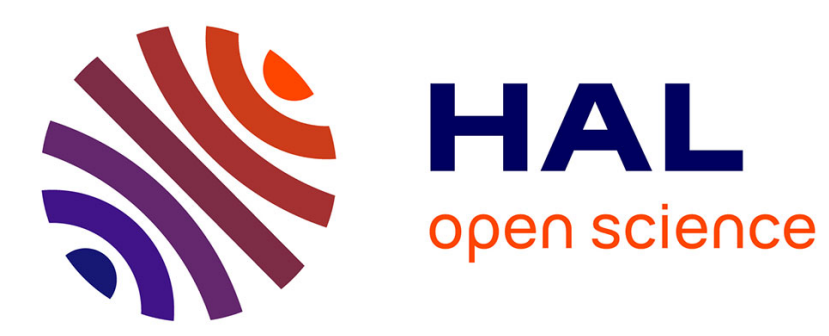

\title{
How can polyreactive antibodies conquer rapidly evolving viruses?
}

\author{
Alejandra Reyes-Ruiz, Jordan D Dimitrov
}

\section{To cite this version:}

Alejandra Reyes-Ruiz, Jordan D Dimitrov. How can polyreactive antibodies conquer rapidly evolving viruses?. Trends in Immunology, 2021, 42 (8), pp.654-657. 10.1016/j.it.2021.06.008 . hal-03344263

\section{HAL Id: hal-03344263 https://hal.sorbonne-universite.fr/hal-03344263}

Submitted on 14 Sep 2021

HAL is a multi-disciplinary open access archive for the deposit and dissemination of scientific research documents, whether they are published or not. The documents may come from teaching and research institutions in France or abroad, or from public or private research centers.
L'archive ouverte pluridisciplinaire HAL, est destinée au dépôt et à la diffusion de documents scientifiques de niveau recherche, publiés ou non, émanant des établissements d'enseignement et de recherche français ou étrangers, des laboratoires publics ou privés. 
How can polyreactive antibodies conquer rapidly-evolving viruses?

\author{
Alejandra Reyes-Ruiz ${ }^{1}$ and Jordan D. Dimitrov ${ }^{1}$ \\ ${ }^{1}$ Centre de Recherche des Cordeliers, INSERM, Sorbonne Université, Université de Paris, \\ 75006 Paris, France
}

\title{
Correspondence to:
}

Jordan D. Dimitrov

Centre de Recherche des Cordeliers

15, rue de l'Ecole de Médecine

75006 Paris - France

E-mail: jordan.dimitrov@sorbonne-universite.fr / jordan.dimitrov@inserm.fr

Tel: +33144278206 


\begin{abstract}
Broadly neutralizing antibodies against rapidly-evolving viruses (e.g. HIV-1 and influenza virus), often manifest antigen-binding promiscuity. Based on a recent study, we hypothesize on the significance of antibody polyreactivity in neutralization of rapidly-evolving viruses. We propose that polyreactivity contributes to tolerate viral variants and shortens time for appearance of neutralizing antibodies.
\end{abstract}

Keywords: antibodies, viruses, influenza virus, HIV-1, polyreactivity, broadly neutralizing antibodies 


\section{Polyreactive antibodies can enable the neutralization of rapidly-evolving viruses}

HIV-1 and influenza A virus (IAV) mutate at a fast pace; this translates into the rapid evolution of envelope proteins (Env) sequences, representing among the principal targets for neutralizing antibody $(\mathrm{Ab})$ responses. The sequence heterogeneity of Env, however, makes the neutralization of rapidly-evolving viruses a daunting task. Typically, Ab responses are limited to viral variants to which the host has already been exposed. In this regard, the ingenious property of the adaptive immune system to differentiate closely-related molecular features might be disadvantageous for the neutralization of rapidly-mutating viruses. Fortunately, the human immune system has preserved a primordial attribute - that of antigenbinding promiscuity, which might be a determining factor for efficient immune responses against pathogens with high genetic variability. Indeed, considerable numbers of Abs capable of neutralizing a broad range of distinct strains of HIV-1 or IAV, broadly neutralizing Abs (bNAbs), display antigen-binding polyreactivity, i.e., the capacity to bind to many virusunrelated antigens in addition to their primary targets $[1,2,3,4]$. However, the question of how polyreactivity can contribute to the broad neutralization of rapidly-evolving viruses remains largely unanswered.

Recently, one study [5] investigated human Ab responses against IAV after natural infection or vaccination. The authors cloned genes encoding variable regions from circulating IAVspecific memory-B cells and plasmablasts to produce panels of recombinant IgG1 Abs. The largest fraction $(>80 \%)$ of these Abs recognized virus Env protein hemagglutinin (HA, BOX 1), and among HA-binding Abs, a significant percentage (42\%) was polyreactive. Of note, $88 \%$ of Abs against the stalk region of HA were polyreactive, as were $95 \%$ of those Abs targeting a broadly neutralizing epitope on this region. In contrast, the fraction of polyreactive Abs binding to the head domain was much lower (28\%) than to the stalk region, implying that Abs directed to the stalk domain, which are frequently endowed with broadly neutralization 
potential, present also higher percentage of polyreactivity, contrary to the Abs directed to head domain and featuring a limited neutralization breadth. The higher preponderance of polyreactivity among IAV stalk-specific Abs agreed with results from other studies [2, 4]. Collectively, these data suggested that Abs binding to conservative sites on HA, i.e., sites vulnerable to neutralization, were predominantly polyreactive [5].

Furthermore, the increased binding of polyreactive Abs to conservative sites on HA resulted in the ability of many of these Abs to bind to distinct strains of IAV H1N1 [5]. The diversity among these strains reflects an evolutionary history of more than 100 years, highlighting the ability of polyreactive Abs to bind to HA, despite marked changes in the sequence. Moreover, polyreactive Abs induced by exposure to human $\mathrm{H} 1$ viruses also recognize zoonotic strains and IAVs belonging to phylogenetically distinct groups, such as type B viruses [5]. In the study, more than half of the polyreactive Abs binding to HA were endowed with neutralization activity; in contrast, only a small fraction of monoreactive Abs exhibited neutralization capacity [5], implying that polyreactivity has functional repercussion for the neutralization of IAV. Also, polyreactive Abs against HA arose predominantly after immunization or infection with novel IAV strains for which the studied individuals were not immune [5], suggesting that the polyreactive Abs developed before the viral-exposure contribute in the immune fight against IAV in nonantigen-experience individuals. Similarly, as in the case of IAV Abs, Abs with broadly neutralizing potential against HIV-1 are frequently ( $>50 \%$ of $\mathrm{Abs}$ ) polyreactive [1], and a direct relationship between polyreactivity and HIV-1-neutralization potency has recently been demonstrated [3]. Taken together these findings imply that polyreactivity directly contributes to broad neutralization of viruses.

In the study discussed above [5], the authors investigated how polyreactive Abs interacted with diverse strains of IAV. As evidenced by molecular dynamics simulations, the antigenbinding sites of polyreactive HA-specific Abs demonstrated increased flexibility relative to 
monoreactive antibodies. This is in accordance with previous reports about increased flexibility in the antigen-binding sites of polyreactive bNAbs against HIV-1 and against other targets compared with binding sites of monoreactive Abs $[3,6,7]$. The variable regions of polyreactive Abs recognizing the HA stalk tend to harbor lower numbers of somatic mutations compared with the variable regions of monoractive HA-binding Abs [5]. Similarly as other polyreactive Abs isolated from healthy individuals or HIV-infected patients, the polyreactive Abs recognizing IAV used with elevated frequency the $\mathrm{V}_{\mathrm{H}} 1-69$ gene, encoding exceptionally hydrophobic CDR H2 loop [5, 9]. Of note, polyreactive Abs achieved broad IAV-recognition potential with a lower number of somatic mutations in comparison to monoreactive Abs, which suggested that these Abs might need shorter times of affinity maturation process for achievement of efficient neutralization of the virus [5]. Similarly, a low number of somatic mutations have been reported for human polyreactive Abs obtained from healthy donors and screened by high-throughput assays [8, 9]. Compelling evidence from X-ray structural and other biophysical analyses shows that the absence or low number of somatic mutations is associated with increased structural dynamics of the Ab antigen-binding sites $[8,10,11]$. In the case of HIV-1 bNAbs however, the elevated conformational flexibility of the paratope, as well as the polyreactivity status, are not explained by the low numbers of somatic mutations [12]. Indeed, these Abs usually harbor abnormally large numbers of mutations and simultaneously manifest polyreactivity. Also, substantial mutation loads, especially in the framework regions of the variable region are known to compromise the protein stability of the $\mathrm{Ab}$, which might result in elevated flexibility of the antigen-binding site relative to the monoreactive Abs specific for the same antigen [12]. Collectively, these findings demonstrate that there are alternative molecular pathways for achievement of enhanced conformational dynamics and polyreactivity of Abs. 


\section{How might Ab polyreactivity contribute to virus neutralization?}

Polyreactive bNAbs against IAV or HIV-1 recognize conservative Env epitopes with high affinity that is commensurate to the affinity of monoreactive bNAbs [2, 3, 5]. Thus, polyreactive bNAbs incongruously incorporate two opposite qualities - stringent binding specificity and binding promiscuity. They may be optimized to bind with high affinity to their target epitope but might also auxiliary interact with other lower affinity antigens because of the enhanced flexibility and better adaptability of their antigen-binding sites to various molecular features on distinct antigens. The findings on bNAbs clearly showed that polyreactivity can be a consequence of the enhanced structural dynamics of antigen-binding site $[5,6,7,10]$. Hence, the precise formulation of the question about the functional role of polyreactivity in broad neutralization of rapidly-evolving viruses should be on how the increased structural dynamics would contribute to the potential of Abs to neutralize a broad range of virus variants.

Considering the structural organization of vulnerable sites on Env - mostly bearing an invariant sequence, and surrounded by regions of high sequence heterogeneity, we proposed the following scenario: if the Ab-binding footprint overlap with the conserved vulnerability site in the antigen without steric effects that can obstruct the access of Abs to its epitope, the broad virus neutralization potential might be achieved by Abs with rigid antigen-binding sites. However, the cognate epitope of the neutralizing Ab might not likely perfectly match the vulnerability site, potentially overlapping molecular surfaces with high variability. Here, certain mutations in the viral protein might compromise $\mathrm{Ab}$ binding at the vulnerability site, perhaps due to steric clashes or incompatibility of electrostatic charges. Also, the large surface area buried by Abs, while binding to protein antigens (ca. $1000 \AA^{2}$ ), might favor simultaneous interactions with both conservative and variable parts of the Env. Thus, the presence of intrinsic conformational dynamics of antigen-binding site might be advantageous 
by giving capacity of Abs to accommodate the disruptive effects of mutations near the site of vulnerability. Of note, analyses of the effect of somatic mutations on the flexibility of some HIV-1 bNAbs suggest that central parts of antigen-binding sites (including CDR H3) are configured early in the affinity maturation process and characterized by structural rigidity [12, 15]. In contrast, somatic mutations might affect the flexibility of polypeptide chains in the periphery of the paratope $[12,15]$. Thus, HIV-1 and IAV bNAbs might use molecular flexibility to overcome the detrimental effect of mutations in regions of little significance for neutralization but which might influence key amino acid residue contacts at sites of vulnerability, although this remains conjectural (Fig. 1a).

Aside from the presumed molecular mechanisms of polyreactive bNAbs, the data from the highlighted study [5] incite speculations regarding the physiological significance of polyreactive Abs in the neutralization of rapidly-evolving viruses; the striking findings that novel strains of IAV can induce broadly reactive polyreactive Abs requiring lower number of somatic mutations to achieve neutralization and broad reactivity might have important repercussions. For instance, the rapid clinical course of IAV infection imposes a requirement for rapid development of neutralizing Abs. Indeed, refined Ab responses with multiple iterations in mutagenesis and B cell selection might be time- and resource-consuming for the host. Hence, Abs polyreactivity can be used as an immune system strategy for 'gaining time': accelerating the evolution of $\mathrm{Ab}$ responses in the race between a virus with ever-evolving antigenic features and the immune system (Fig. 1b). Further investigations regarding the mechanisms leading to selection and production of polyreactive bNAbs might have significant importance in the development of vaccines against viruses with high genomic diversity. 


\section{Acknowledgments}

This work was supported by Institut National de la Santé et de la Recherche Médicale (INSERM, France). A.R.R. is recipient of fellowship from an Innovative Training Network (ITN) funded by the European Union's Horizon 2020 Programme under grant agreement No 859974, project EDUC8. J.D.D. is recipient of a grant from the European Research Council (Project CoBABATI ERC-StG-678905). We are grateful to Dr Sebastien Lacroix-Desmazes (Centre de Recherche des Cordeliers, Paris) for reading and fruitful discussion about the manuscript.

\section{References}

1. Liu, M. et al. (2015) Polyreactivity and autoreactivity among HIV-1 antibodies. $J$. Virol. 89, 784-798.

2. Andrews, S.F. et al. (2015) Immune history profoundly affects broadly protective B cell responses to influenza Sci. Transl. Med. 7, 316ra192.

3. Prigent, J. et al. (2018) Conformational Plasticity in Broadly Neutralizing HIV-1 Antibodies Triggers Polyreactivity. Cell Rep. 23, 2568-2581.

4. Bajic, G. et al. (2019) Autoreactivity profiles of influenza hemagglutinin broadly neutralizing antibodies Sci. Rep. 9, 3492.

5. Guthmiller, J.J. et al. (2020) Polyreactive Broadly Neutralizing B cells Are Selected to Provide Defense against Pandemic Threat Influenza Viruses Immunity 53, 12301244.e1235.

6. Finton, K.A.K. et al. (2014) Ontogeny of recognition specificity and functionality for the broadly neutralizing anti-HIV antibody 4E10 Plos Pathog. 10, e1004403.

7. Adhikary, R. et al. (2015) Adaptive mutations alter antibody structure and dynamics during affinity maturation Biochemistry 54, 2085-2093.

8. Shehata, L. et al. (2019) Affinity Maturation Enhances Antibody Specificity but Compromises Conformational Stability. Cell Rep. 28, 3300-3308 e3304.

9. Boughter, C.T. et al. (2020) Biochemical patterns of antibody polyreactivity revealed through a bioinformatics-based analysis of CDR loops eLife 9, e61393. 
10. Manivel, V. et al. (2000) Maturation of an antibody response is governed by modulations in flexibility of the antigen-combining site. Immunity 13, 611-620.

11. Fernández-Quintero, M.L. et al. (2020) Local and Global Rigidification Upon Antibody Affinity Maturation Front. Mol. Biosci. 7, 182.

12. Klein, F. et al. (2013) Somatic mutations of the immunoglobulin framework are generally required for broad and potent HIV-1 neutralization. Cell 153, 126-138.

13. Ovchinnikov, V. et al. (2018) Role of framework mutations and antibody flexibility in the evolution of broadly neutralizing antibodies eLife 7, e33038.

14. Henderson, R. et al. (2019) Selection of immunoglobulin elbow region mutations impacts interdomain conformational flexibility in HIV-1 broadly neutralizing antibodies Nat. Commun. 10, 654.

15. Davenport, T.M. et al. (2016) Somatic Hypermutation-Induced Changes in the Structure and Dynamics of HIV-1 Broadly Neutralizing Antibodies Structure 24, 1346-1357.

\section{BOX 1 Immunogenicity of hemagglutinin}

Influenza virus hemagglutinin is divided in two structural and functional domains - head and stalk. The head domain contains a receptor-binding site, whereas the stalk domain is responsible for the fusion of viral membrane with the cell membrane. The head region is immunodominant i.e. most of $\mathrm{Ab}$ responses are directed towards it. However, in the largest part, these responses are focused to highly variable regions of the domain and therefore they have only a limited neutralization breadth. Contrary to the Abs binding to head domain, Abs recognizing stalk, especially a particular conservative epitope on it, are frequently endowed with a broadly neutralizing potential. 


\section{Figure legend}

Figure 1. Human polyreactive antibodies and virus neutralization. a. A virus-neutralizing antibody with rigid antigen-binding site recognizes the site of vulnerability of virus strain A, which will result in neutralization. Variations in the amino acid sequence surrounding the vulnerability site in virus strain B can prevent the antibody with rigid antigen-binding site to contact the site of vulnerability and thus the virus evades neutralization (Left panel). A polyreactive virus-neutralizing antibody has flexible antigen-binding sites. The molecular flexibility would allow the antibody to overcome constrains due to variation of the Env sequence surrounding the invariant site of vulnerability, thus antibody would be able to access to the site of vulnerability and neutralize both viral strains (A and B) (Right panel). b. Suggested role of antibody polyreactivity in immune responses against influenza virus. B cells expressing BCR with rigid antigen-binding site would require higher number of iterations of somatic mutations and selection during affinity maturation process before producing an antibody capable to neutralize the virus. A B cell clone expressing BCR with flexible antigen-binding site will reach a state of efficient neutralization of broader range of influenza virus strains after lower number of iterations during affinity maturation process. 
a

$\mathrm{Ab}$ with rigid antigen-binding site
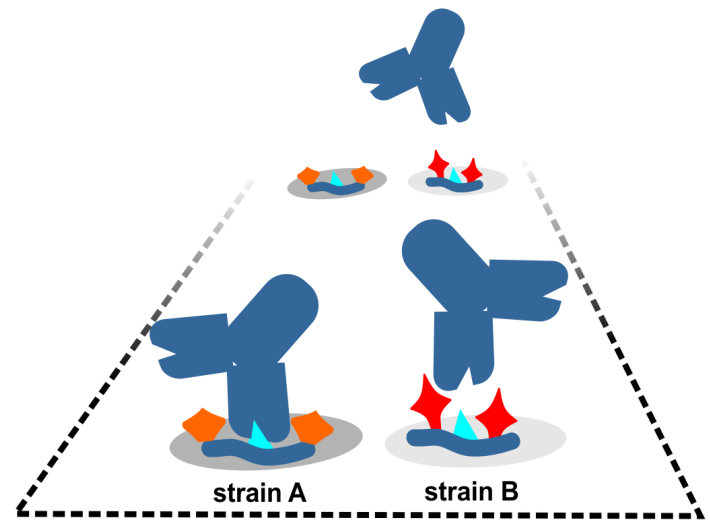

1 variable sites
$A b$ with flexible antigen-binding site

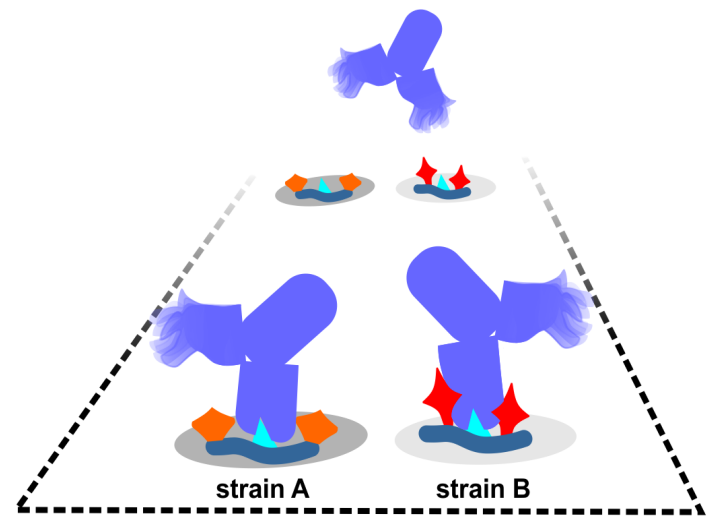

site of vulnerability

b

Affinity maturation iterations

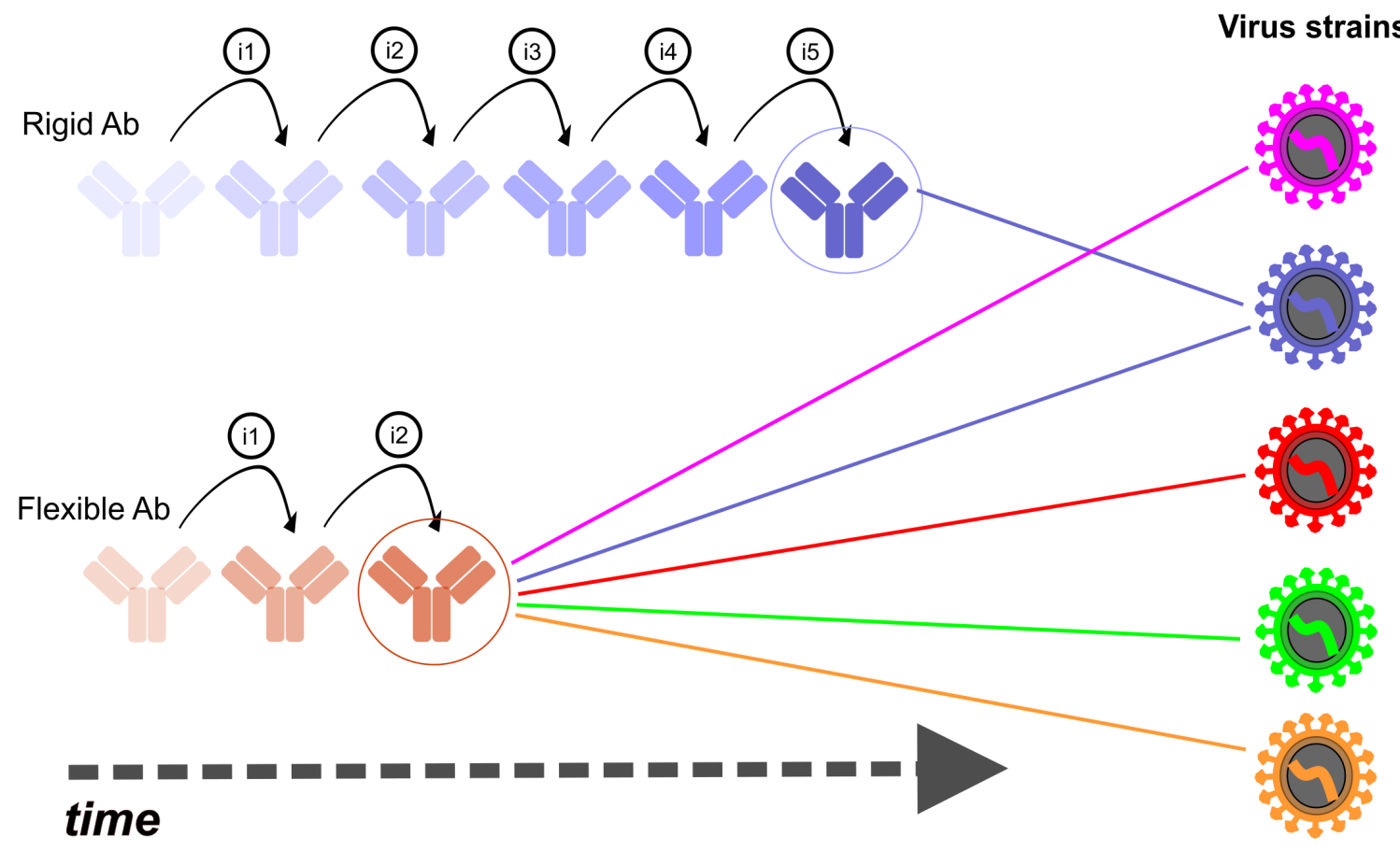

